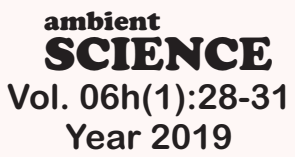

ORIGINAL ARTICLE

\title{
Comparative Evaluation on the Therapeutic Outcome of Minimally Invasive Plating Osteosynthesis Method with Open Reduction and Internal Fixation for Treatment of Distal Tibial Fractures
}

\author{
Ahmad Dasht Bozorg, Milad Heidarian* \\ Department of Orthopaedics and Traumatology, Jundishapur \\ University of Medical Sciences, Ahvaz, Iran \\ Study Area: Ahvaz, Iran \\ Coordinates:31 $19^{\prime} 13^{\prime \prime N} ; 48^{\circ} 40^{\prime} 09^{\prime \prime E}$
}

Key words: ORIF, MIPO, AOFAS Index

This study is approved by the Ethics committee of the Jundishapur University of Ahvaz (Code: IR.AJUMS.REC.1397.834).

\section{Introduction:}

Tibia is one of the most important bones that is fractured with severe soft tissue damage and is subject to fracture complications. plastering, pin and plaster technique, Open Reduction and Internal Fixation (ORIF), Intramedullary Nailing and External Fixator, are some of the common treatments for these types of fractures which have specific indications and side effects (Rudloff, 2013; Court-Brown, 2006). Minimally invasive plating osteosynthesis (MIPO) or Bridging Plate is somehow a new method that has been mentioned in scientific papers in recent years (Miclau \& Martin, 1997; Krettek, 1997; Baumgaertel et al., 1998; Perren, 2001). Nowadays, less invasive methods are more widely considered due to less damage to soft tissues, the hematoma of the fracture site and the periosteum. The basis of this new method is to maintain the hematoma of the fracture and to create the proper situation for the establishment and development of the primary bone marrow, which leads to an

\section{Abstract}

Tibial fractures with soft tissue injury, lead to disability for a long time. The study aimed to compare the success rate of Minimally Invasive Plating Osteosynthesis (MIPO) with the outcomes of Open Reduction and Internal Fixation (ORIF) method in the treatment of tibia fractures. This retrospective cross-sectional study was conducted on 43 patients with distal tibia fractures treated either of two methods ORIF or MIPO . In addition to evaluating the feasibility of MIPO technique in the tibia, the duration of union and organ function in daily activities were also evaluated. There was no significant difference in the mean union time, the mean time of return to previous activity and The American Orthopaedic Foot and Ankle Society (AOFAS) score between the two groups. But, the incidence of wound complications, as well as soft tissue damage, was significantly higher in the ORIF group. The prevalence rate of infection at the site of operation was also significantly higher in the ORIF group. Despite better results in the MIPO group, there was no significant difference between the two methods in terms of clinical improvement and the functional score of patients, and in the tibia fractures with the type of $A$ and $B$, they were not superior to each other. The lower rates of complications in the MIPO group evidenced it as a useful and practical technique.

increase in the rate of union and accelerates it, as well as to the reduction of complications (Farouk et al., 1997). Impact of tibia fractures cause disability for a long time, especially in young people which are effective forces of society; hence their treatment has always been a major concern for the community of orthopedic surgeons. The purpose of this study was to determine the success rate of the MIPO technique as a new method and compare with the results of ORIF method to help achieve an optimal method for treating tibia fractures.

\section{Methodology:}

This is a retrospective cross-sectional study conducted in the year 2016-2017 on patients aged 18-6o years suffering from distal tibia fractures types $\mathrm{A}, \mathrm{B}$ according to the $\mathrm{AO}$ OTA classification, which was treated with either ORIF or MIPO method at Razi Hospital of Ahvaz. First of all the patients were divided into two groups; ORIF $(\mathrm{n}=25)$ and 


\section{ORIGINAL ARTICLE}

MIPO $(n=18)$, then the Patients with at least 6 months and up to 2 years follow up duration were selected for attending the clinic and conducting clinical and radiographic assessments. In the MIPO group, from 22 selected patients, 2 patients were excluded due to their lack of willingness to participate in the study, 1 patient due to incomplete postoperative control graphs, and 1 patient due to death was excluded from the study, and finally, the treatment results of 18 eligible patients were evaluated.

The fracture stabilization device in the MIPO group was distal tibia anatomical locked plate. Patients were examined separately by the resident and the orthopedic specialist. In addition to evaluating the MIPO technique, the duration of union and organ function in daily activities were also evaluated. Patients with more than 2 weeks between fracture and surgery time, patients with fractures in other organs, patients with an open fracture (except Gustilo Type I), patients with head trauma hospitalized in the intensive care unit, patients residing in other cities and burned in the same limbs were excluded from the study. Eligible patients were informed after receiving written consent. In the last follow up, the time of union, the rate of complete union and delay union were recorded in the two groups. In order to evaluate the beginning of welding and welding completely, control radiographs were performed routinely at patients' intervals of 4 weeks, 6 weeks, 3 months and 6 months after surgery. Functional outcome was evaluated using The American Orthopaedic Foot and Ankle Society (AOFAS) scoring criterion. AOFAS score is in the range of $\mathrm{o}-100$ and the classification is "excellent" (100-90), "good" (89-80), "fair" (79-6o), and "poor" (less than 6o). Independent t-test and Chi-square test were used to evaluate and compare the quantitative and qualitative data, respectively. Data were analyzed using SPSS22 software and P-value<0.05 was considered as the signif icance level.

\section{Results:}

No significant difference was revealed between the mean age of the patients in both groups at $5 \%$ level. In both groups, the number of male patients was higher than female, but regarding the sexual distribution, two groups were consistent with each other and there was no significant difference between them at $5 \%$ level. There was no significant difference at the $5 \%$ level between the distribution of patients according to the type of fracture. In both groups, the right fracture was more prevalent than the left side, but there was no signif icant difference between the distribution of patients according to the fracture site in the two groups at $5 \%$ level. The difference of mean duration of follow-up was not statically significant between the two groups.

There was no significant difference between mean union time in the two groups. In the MIPO group, 3 patients with delayed and nonunion fracture underwent revision surgery by bone graft. From 15 patients with a complete
Ambient Science, 2019: Vol. 06h(1); 28-31 DOI:10.21276/ambi.2019.06h.1.oa06

union, 12 patients have removed the device a few months before the latest follow up (mean 6 months), and evaluations were performed for these patients based on the postoperative control graphs. In the ORIF group, only one patient with nonunion fracture underwent revision surgery by bone graft. Although the rate and time of fracture union were better in the MIPO group, the percentage of patients requiring revision surgery and bone graft in this group was greater than the ORIF group $(16.67 \%$ vs. $4 \%)$. All the patients with delayed or nonunion in two groups had also fracture in the fibula. The incidence of wound complications, as well as soft tissue damage, was significantly higher in the ORIF group $(\mathrm{p}<0.05)$. The incidence rate of infection at the site of operation was significantly higher in the ORIF group $(\mathrm{p}<0.05)$. None of the patients with infection was reported in the MIPO group. The mean duration of return to previous activities in the ORIF group was lower than the MIPO group, but the difference was not statistically significant between the two groups. Although the mean functional score in AOFAS score was higher in MIPO group, there was no significant difference between the two groups in the mean score of AOFAS and based on the classification in this criterion, functional scores in both the groups was placed in the "good" level (Table-2).

Table 1. Demographic and clinical information of patients in two groups

\begin{tabular}{|c|c|c|c|c|}
\hline \multicolumn{2}{|l|}{ Variable } & $\operatorname{MIPO}(\mathrm{n}=18)$ & ORIF $(n=25)$ & p value \\
\hline \multicolumn{2}{|c|}{ Age (yr) (Mean $\pm S D)$} & $\begin{array}{l}38.61 \pm 7.12 \\
(27-56)\end{array}$ & $\begin{array}{l}32.28 \pm 9.07 \\
(18-58)\end{array}$ & $>0.091$ \\
\hline Gender & Women & $4(22.22 \%)$ & $8(32 \%)$ & $>0.082$ \\
\hline$(\mathrm{N}, \%)$ & Men & $14(77.78 \%)$ & $17(68 \%)$ & \\
\hline Fracture type & A & $8(44.44 \%)$ & $10(40 \%)$ & $>0.74$ \\
\hline$(\mathrm{N}, \%)$ & B & $11(61.11 \%)$ & $15(60 \%)$ & \\
\hline Location & Right & $12(66.67 \%)$ & $13(52 \%)$ & $>0.12$ \\
\hline$(\mathrm{N}, \%)$ & Left & $6(33 \cdot 33 \%)$ & $7(28 \%)$ & \\
\hline $\begin{array}{l}\text { Follow-up tim } \\
(\text { Mean } \pm \text { SD) }\end{array}$ & (month) & $16.01 \pm 2.44$ & $15.27 \pm 1.72$ & $>0.84$ \\
\hline
\end{tabular}

Table 1. Demographic and clinical information of patients in two groups

\begin{tabular}{|c|c|c|c|}
\hline Variable & $\operatorname{MIPO}(n=18)$ & ORIF $(n=25)$ & p value \\
\hline $\begin{array}{l}\text { Bone union time (week) } \\
(\text { Mean } \pm \text { SD) }\end{array}$ & $19.12 \pm 2.17$ & $20.17 \pm 3.88$ & $>0.52$ \\
\hline Rate of Union (N, \%) & $15(83.33 \%)$ & $19(76 \%)$ & $>0.92$ \\
\hline Malunion (N, \%) & $\mathrm{o}(\mathrm{o} \%)$ & $1(4 \%)$ & \\
\hline Nonunion (N, \%) & $1(5 \cdot 56 \%)$ & $1(4 \%)$ & \\
\hline $\begin{array}{l}\text { Soft tissue irritation } \\
(\mathrm{N}, \%)\end{array}$ & $1(4 \%)$ & $4(22.22 \%)$ & $<0.002$ \\
\hline $\begin{array}{l}\text { Wound complications } \\
(\mathrm{N}, \%)\end{array}$ & $1(4 \%)$ & $3(16.67 \%)$ & $<0.031$ \\
\hline Infection (N, \%) & o $(0 \%)$ & $3(12 \%)$ & $<0.001$ \\
\hline $\begin{array}{l}\text { Time of recovery to work } \\
\text { (week) } \\
(\text { Mean } \pm \text { SD) }\end{array}$ & $23.27 \pm 2.07$ & $21.03 \pm 2.11$ & $>0.45$ \\
\hline AOFAS Index (Mean \pm SD) & $88.23 \pm 10.4$ & $86.70 \pm 10.37$ & $>0.42$ \\
\hline
\end{tabular}




\section{Discussion:}

We compared the outcomes of MIPO and ORIF methods in the treatment of tibia fracture in terms of union rate and surgical complications. No significant differences, between mean age, distribution of gender, fracture type and distribution of patients according to the involved side in two groups were noticed and they were consistent with each other in demographic and clinical factors.

There was no statistical difference between the mean time of union fracture in the two groups (20.17 weeks in the ORIF group and 19.29 weeks in the MIPO group) ( $p>0.05$ ). In the study of Kim et al. (2018) all fractures were a union without the need for re-intervention, the mean union time in the MIPO group was 17.4 weeks and in the minimal ORIF group was 16.3 weeks, which was less than the reported period in our study, but they were consistent with no significant differences in the results between two groups. Further, in the study of Fang et al. (2016) the time of union formation was not statistically different in the study groups ( $p>0.05)$. In their study, patients in the EF + LORIF group needed more secondary interventions after the first surgery, mainly due to the occurrence of pin and component infections (14.3\%). In the study of Devkota et al. (2014) the mean rate of union formation in the MIPO group was 25 weeks ( 20 to 30 weeks), which was higher than the reported rate in the present study. In their study, in all patients but no 2 cases, complete union fracture was performed that was consistent with the results of the current study.

We found the incidence of wound complications, as well as soft tissue damage in the ORIF group, was statistically higher $(22.22 \%$ vs. $4 \%)(\mathrm{p}<0.05)$ which contradict with the study of Kim et al. (2018). In Fang et al. (2016), skin and wound complications resulting from surgery in the MIPO group were significantly more than EF+LORIF, which was not consistent with the results of our study. In the present study, the prevalence rate of infection at the site of operation was statistically higher in the ORIF group $(12 \%)(\mathrm{p}<0.05)$. The incidence of infection were reported in none of the patients in the MIPO group. In the study of Kim et al. (2018) two groups were equal in terms of the incidence of infection. In the study of Fang et al. (2016) for EF + LORIF group, patients mostly needed secondary interventions due to infection caused by pin and plate, which was consistent with our present study in the higher rate of infection in the ORIF group. In a study by Devkota et al. (2014), 5 patients had a superficial infection in the in MIPO group, which their results were not consistent with the present study.

The mean duration for returning to the previous activities in ORIF group was lower than the MIPO group, but statistically, this time was not significantly different between the two groups. In the study of Fang et al. (2016), there was no significant difference between the time to return to previous activity in the EF+LORIF and MIPO group, which was consistent with the results of the present study. Although the mean score of AOFAS was higher in patients with MIPO group (88.23 Vs. 86.07), the mean score of AOFAS in both groups was not signif icantly different and based on the classification of AOFAS, the mean scores of both groups were in "good" level. In the study of Kim et al. (2018), there was no statistical difference between the AOFAS score in the MIPO group (86) and the minimal ORIF group (86.7), which was consistent with the results of our present study. In the study of Fang et al. (2016) the mean score of AOFAS was not significantly different in the study groups which was consistent with the results of the present study. In the study of Cheng et al. (2011) where the Mazur scoring criterion was used to evaluate the patient's postoperative performance, in the ORIF group, the results of 10 patients (Excellent), 3 patients (Good), and 1 patient (Fair) and in the MIPO group were 1o Patient (Excellent) and 5 Patients (Good), which was in agreement with our present study in terms of better performance score in the MIPO group.

The limitations of our study were surgeons diversity due to the location of the research, which was a universityaff iliated educational hospital, as well as retrospective and inaccessible to all treated patients, especially in the MIPO group. In future studies, designing prospective studies with a higher sample size and performing all surgeries by an expert surgeon are recommended.

Conclusively, despite the better results in the MIPO group, there were no significant differences between the two methods in terms of clinical improvement and functional score, and in tibia fractures (type A, B), they were not superior to each other. The lower rates of complications in the MIPO group show the usefulness and utility of this technique. On the other hand, the MIPO surgery technique is simpler than other methods and does not require very advanced equipment and can be done in most hospital centers even in deprived areas. Meanwhile, the necessary equipment for surgery in the MIPO method is cheaper than other methods.

\section{Acknowledgment:}

We are thankful to all particinats who had allowed us to carried out this study on their infant. We also thankful to the other medical staffs for helping us in various ways to conduct this study

\section{References:}

Baumgaertel, F., Buhl, M. \& Rahn, B. (1998): Fracture healing in biological plate osteosynthesis. Injury, 29(Suppl 3):C3-6.

Court-Brown, C.M. (2006): Fractures of the tibia and fibula, pp. 1845-1914. In: Rockwood, C.A.Jr, Green, D.P., Bucholz, R.W. (eds.), Rockwood \& Green's Fractures in Adults. Pub. by: Lippincott Williams \& Wilkins, Philadelphia.

Cheng, W., Li, Y., Manyi, W.(2011): Comparison study of two surgical options for distal tibia fracture-minimally invasive plate osteosynthesis vs. open reduction and internal fixation. Int. Orthop., 35(5):737-742.

Devkota, P., Khan, J.A., Shrestha, S.K., Acharya, B.M., Pradhan, N.S., Mainali, L.P., Khadka, P.B. \& Manandhar, H.K. (2014): 


\section{ORIGINAL ARTICLE}

Minimally invasive plate osteosynthesis for distal tibial fractures. J. Orthop. Surg., 22(3):299-303.

Fang, J.H., Wu, Y.S., Guo, X.S. \& Sun, L.J. (2016): Comparison of 3 minimally invasive methods for distal tibia fractures. Orthopedics, 39(4):e627-633.

Farouk, O., Krettek, C., Miclau, T., Schandelmaier, P., Guy, P. \& Tscherne, H. (1997): Minimally invasive plate osteosynthesis and vascularity: preliminary results of a cadaver injection study. Injury, 28(Suppl-1):A7-12

Kim, J.W., Kim, H.U., Oh C-W, Kim, J-W \& Park, K.C. (2018): A Prospective Randomized Study on Operative Treatment for Simple Distal Tibial Fractures-Minimally Invasive Plate Osteosynthesis Versus Minimal Open Reduction and Internal Fixation. L. Orthop. Trauma, 32(1):e19-e24.
Ambient Science, 2019: Vol. 06h(1); 28-31 DOI:10.21276/ambi.2019.06h.1.oa06

Krettek, C. (1997): Foreword: concepts of minimally invasive plate osteosynthesis. Injury, 28(Suppl 1):A1-A2.

Miclau, T. \& Martin, R. (1997): The evolution of modern plate osteosynthesis. Injury, 28(Suppl 1):A3-A6.

Rudloff, M.I. (2013): Fractures of Lower Extremity, pp. 2617-2720. In, Canale, S.T. \& Beaty, J (eds.), Campbelle's Operative Orthopedics (Vol. III). Pub. by: Mosby, an imprint of Elsevier Inc., Philadelphia.

Perren, S.M. (2001): Minimally invasive internal fixation: History, essence and potential of a new approach. Injury, 32 (Suppl 1):A1-A3. 